\title{
Accurate Parametric Modeling of Folded Waveguide Circuits for Millimeter-Wave Traveling Wave Tubes
}

\author{
John H. Booske, Senior Member, IEEE, Mark C. Converse, Student Member, IEEE, \\ Carol L. Kory, Senior Member, IEEE, Christine T. Chevalier, Student Member, IEEE, David A. Gallagher, \\ Kenneth E. Kreischer, Member, IEEE, Vernon O. Heinen, Member, IEEE, and Sudeep Bhattacharjee, Member, IEEE
}

\begin{abstract}
In this paper, results of different models are compared for calculating effective, cold-circuit (beam-free) phase velocities and interaction impedances of folded waveguide (FW) slow wave circuits for use in millimeter-wave traveling wave tubes (TWT). These parameters are needed for one-dimensional (1-D) parametric model simulations of FW traveling wave tubes (FWTWTs). The models investigated include approximate analytic expressions, equivalent circuit, three-dimensional (3-D) finite difference, and 3-D finite element. The phase velocity predictions are compared with experimental measurements of a representative FW circuit. The various model results are incorporated into the CHRISTINE1D code to obtain predictions of small signal gain in a 40-55 GHz FWTWT. Comparing simulated and measured frequency-dependent gain provides a sensitive, confirming assessment of the accuracy of the simulation tools. It is determined that the use of parametric 1-D TWT models for accurate, full band predictions of small signal gain in FWTWTs requires knowledge of phase velocity and impedance functions that are accurate to $<0.5 \%$ and $<10 \%$, respectively. Saturated gain predictions, being approximately half as sensitive to these parameters, appear to require correct specification of phase velocity and interaction impedance to within $\sim 1 \%$ and $20 \%$, respectively. Although all models generate sufficiently accurate predictions of the interaction impedance, not all generate sufficiently accurate predictions of the effective axial phase velocity.
\end{abstract}

Index Terms-Folded waveguide, millimeter-wave, numerical simulation, traveling wave tube (TWT).

\section{INTRODUCTION}

$\mathbf{E}$ MERGING applications in electronic counter measures, radar, communications, and imaging require lightweight, low voltage $(\leq 12 \mathrm{kV})$, compact and broadband $(\Delta f / f>10 \%)$ sources of high average power $(>100 \mathrm{~W})$ at millimeter-wave frequencies $(f \sim 30-100 \mathrm{GHz})$. The folded waveguide traveling wave tube (FWTWT) [1] is an excellent candidate for these applications. This device uses an electric field plane $(E$ plane) bend serpentine rectangular waveguide as a slow wave circuit, as illustrated in Fig. 1. A linear electron beam passing through small holes in the broad wall of a rectangular waveguide

Manuscript received July 27, 2004. The review of this paper was arranged by Editor W. L. Menninger.

J. H. Booske, M. C. Converse, and S. Bhattacharjee are with the Department of Electrical and Computer Engineering, University of Wisconsin-Madison, Madison, WI 53706 USA (e-mail: booske@engr.wisc.edu).

C. L. Kory and C. T. Chevalier are with the Analex Corporation, NASA Glenn Research Center, Cleveland, OH 44135 USA.

D. A. Gallagher, K. E. Kreischer, and V. O. Heinen are with the Northrop Grumman Corporation, Rolling Meadows, IL 60008-1098 USA.

Digital Object Identifier 10.1109/TED.2005.845798 interacts with the colinear electric field of a propagating, fundamental $\mathrm{TE}_{10}$ mode. The polarity of the electric field reverses at each $E$-plane bend with respect to the electron beams velocity vector [2]. Hence, the FW circuit belongs to the same "fundamentally backward" class of circuits as the coupled cavity TWT (CCTWT).

Design and analysis of TWTs has been greatly facilitated in the last few years by the emergence of new computational software tools [3]. These include generic electromagnetic problem solvers and TWT-specific models [4]-[7], [14]. To date, most of the analysis and experimental validation studies have focused on helix-TWTs, and to a lesser extent, CCTWTs.

As a general rule, helix-TWTs are capable of the largest bandwidths, but FWTWTs and CCTWTs provide higher average power handling capability. Compared to conventional CCTWTs, FWTWTs can provide larger bandwidth (20\%-30\% versus $10 \%-15 \%$ ). In principle, FWTWT circuits are also easier to fabricate than conventional CCTWT circuits. This latter feature has motivated an investigation of the FWTWT as a compact coherent source for the submillimeter or terahertz regime [8], [9].

Because of near-symmetry in the azimuthal dimension, helixTWTs can be simulated with one-, two-, and three-dimensional models. In contrast, FWTWTs have no azimuthal symmetries and must therefore be either modeled in one or three dimensions. Three-dimensional (3-D) models have the attraction of being highly physical, and can account for complex configuration effects. However, they can be very time and memory intensive, and are therefore of limited utility in early stages of new device design. One-dimensional (1-D) parametric codes [6], on the other hand, have been developed to a sufficient level of sophistication as to very accurately predict numerous features of helix-TWT performance. A review and comparison of helix-TWT and FWTWT basic physics [2] leads one to expect equally accurate 1-D simulations of FWTWTs, provided appropriate and accurate characterizations are obtained for the frequency-dependent effective phase velocity and interaction impedance parameter functions. With reference to Fig. 1

$$
v_{\mathrm{ph}, m}=\frac{\omega}{\frac{\Delta \phi_{z, m}}{p}}
$$

is the effective axial phase velocity of the $m$ th spatial harmonic. $\omega$ is the angular frequency of the wave ( $\mathrm{rad} / \mathrm{s}) \Delta \phi_{z, m}$ is the phase difference of the $m$ th spatial harmonic's axial electric 


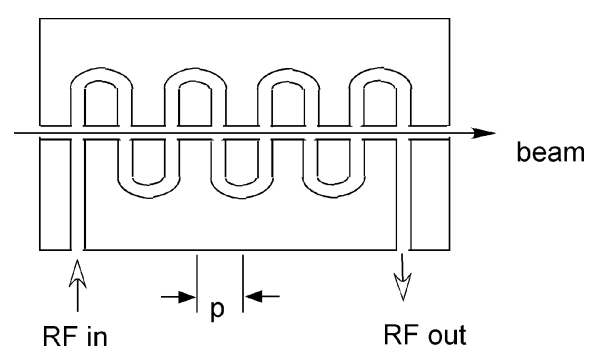

Fig. 1. Illustrative sketch of a FW TWT slow-wave circuit.

field $E_{z, m}$ between subsequent beam crossings of the waveguide and $p$ is the distance between subsequent beam crossings, or the "pitch" of the circuit.

$$
K_{\mathrm{axis}, m}=\frac{\left|E_{z, m}\right|^{2}}{2 \beta_{z, m}^{2} P_{\mathrm{wg}}}
$$

is the effective, on-axis interaction impedance of the $m$ th spatial harmonic. $P_{\mathrm{wg}}$ represents the power flowing in the $\mathrm{TE}_{10}$ mode associated with a particular on-axis, longitudinal electric field strength $E_{z, m}$ and $\beta_{z, m}$ is the effective axial wavenumber for the $m$ th spatial harmonic

$$
\beta_{z, m}=\frac{\Delta \phi_{z, m}}{p}
$$

Proper analysis requires determination of these parameters for the space harmonic that is synchronous with the electron beam, which in this case is $m=0$, as discussed in [2].

The types of applications envisaged for FWTWTs (compact, higher average power) lead to large space charge beams. Consequently, accurate design and analyses with 1-D parametric TWT models are very sensitive to accurate specification $(<$ $0.5 \%$ error) of the frequency-dependent effective phase velocity function, $v_{\mathrm{ph}, m}(\omega)$. Accurate knowledge of $K_{\mathrm{axis}, m}(\omega)$ is also important, but not as critical as $v_{\mathrm{ph}, m}(\omega)$. This issue will be discussed in later sections.

In the remainder of this paper, the predictions of $v_{\mathrm{ph}, m}(\omega)$ and $K_{\text {axis, } m}(\omega)$ are described and compared, using approximate analytic expressions, equivalent circuit, 3-D finite difference, and 3-D finite element models. The phase velocity predictions are compared with experimental measurements of a representative FW circuit. The various model results are also incorporated into the CHRISTINE1D code to obtain predictions of small and large signal gain in a $40-55 \mathrm{GHz} 100-\mathrm{W}$ FWTWT [10]. It is shown that comparing the gain predictions with experimental measurements of frequency-dependent gain provides a sensitive, confirming assessment of the predicted parameters' accuracy. More importantly, these simulations establish that small signal gain predictions are more sensitive than saturated gain predictions to the accuracy of either parametric function. Moreover, the small signal gain predictions are significantly more sensitive to the accuracy of the phase velocity than the interaction impedance.

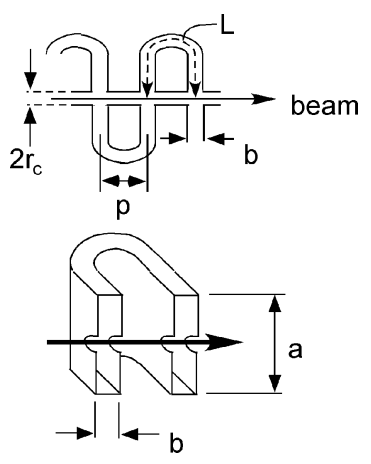

Fig. 2. FW circuit sketch comparing $L$ and $p$, the wave and beam pathlengths, respectively, between beam-crossings. Also indicated are the transverse dimensions, $a$ and $b$ of the rectangular guide, and the beam hole radius, $r_{c}$.

\section{DESCRIPTION OF THE MODELS AND EXPERIMENTAL METHODS}

\section{A. Analytic Models}

For a smooth-wall FW circuit, the apparent axial electric field's phase shift between beam-crossings for the $m$ th spatial harmonic can be estimated to be

$$
\Delta \phi_{z, m}=\left[\left(\frac{\omega}{v_{z, \text { wave }}}\right) p+\pi\right]+2 \pi m .
$$

Here, $v_{z, \text { wave }}$ is the axial phase velocity of the wave [2]

$$
v_{z, \text { wave }}=\frac{p}{L} \frac{c}{\sqrt{1-\left(\frac{\omega_{c o}}{\omega}\right)^{2}}}
$$

where $c$ is the speed of light, $\omega_{\mathrm{co}}$ is the cutoff frequency for the $\left(\mathrm{TE}_{10}\right)$ propagating mode

$$
\omega_{\mathrm{co}}=\frac{\pi c}{a}
$$

$L$ is the path length for the wave between beam-crossings, as shown in Fig. 2, and $a$ is the larger transverse dimension of the rectangular waveguide.

Substitution of (4)-(6) into (1) provides an analytic estimate for the FW circuits effective axial phase velocity seen by the electrons in the beam

$$
v_{\mathrm{ph}, m}=\frac{p}{L} \frac{c}{\sqrt{1+\left(\frac{\omega_{\mathrm{co}}}{\omega}\right)^{2}}+\frac{\pi c}{\omega L}+\frac{2 \pi m c}{\omega L}} .
$$

This model does not account for the effects of the circuit bends and beam tunnel holes

Note that, $\nu_{z \text {,wave }}((5))$ is the projected axial wave velocity of the wave, whereas $\nu_{\mathrm{ph}, m}[(7)]$ is the effective axial phase-advance velocity seen by the electrons. The electrons see an additional $180^{\circ}$ phase shift in the wave, based upon the physical structure of the FW and the beam hole. These two velocities are similar. In fact, the only difference results from the incorporation of this $180^{\circ}$ phase shift phenomenon. 


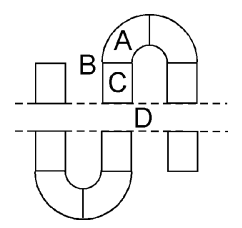

Fig. 3. Sketch of a section of the FW circuit indicating the basic components. (A) $E$-plane circular bend. (B) Circular bend - straight waveguide junction. (C) Straight waveguide section. (D) Electron beam hole.

An analytic estimate for the on-axis interaction impedance can also be obtained, following the derivation in [2] but accounting for waveguide cutoff effects. The result is

$$
\begin{aligned}
K_{\mathrm{axis}, m} & =K_{\mathrm{wg}, 10} \frac{1}{\left(\beta_{z, m} p\right)^{2}}\left[\frac{\sin \left(\frac{\beta_{z, m} b}{2}\right)}{\frac{\beta_{z, m} b}{2}}\right]^{2} \frac{1}{I_{0}^{2}\left(\kappa_{\mathrm{cm}} r_{c}\right)} \\
K_{\mathrm{wg}, 10} & =\frac{2 b}{a} \frac{\eta_{0}}{\sqrt{1-\frac{\omega_{\mathrm{co}}^{2}}{\omega^{2}}}} \\
\kappa_{\mathrm{cm}}^{2} & =\beta_{z, m}^{2}-\left(\frac{\omega}{c}\right)^{2}
\end{aligned}
$$

where $r_{b}$ is the beam radius, $\eta_{0} \approx 377 \Omega$ is the "impedance of free space" and $K_{\mathrm{wg} 10}$ is the waveguide impedance of the $\mathrm{TE}_{10}$ mode. $I_{0}$ is a modified Bessel function.

\section{B. Equivalent Circuit Model}

Another method of determining the phase velocity of a FW is with an equivalent circuit model. The different components of the FW are represented as circuit components, each with their own transmission line transfer matrix. The composite FW is modeled by serial multiplication of the individual transfer matrices into a single, cascaded, or composite transmission matrix. Fig. 3 shows a sketch of a section of a FW. A, B, C, and D designate the critical components: $E$-plane circular bend, circular bend - straight waveguide junction, straight waveguide section, and electron beam hole, respectively.

From the "electron beam's perspective," there is just a net phase advance between subsequent crossings of the waveguide. To mathematically describe the effective dispersion properties of this circuit from the electron beam perspective, one would equate the multicomponent cascaded transmission matrix to a single transmission matrix constructed by treating the entire FW as a single transmission line segment [11]. Since the former (cascaded) matrix is solvable, the characteristics of the latter single equivalent matrix can be determined.

For the single equivalent matrix, we can represent the fields in the FW as a transmission line [11], where

$$
\begin{aligned}
V\left(z_{o}+p\right) & =V\left(z_{o}\right) \cos (\kappa)+j Z I\left(z_{o}\right) \sin (\kappa) \\
I\left(z_{o}+p\right) & =I\left(z_{o}\right) \cos (\kappa)+j Y V\left(z_{o}\right) \sin (\kappa) .
\end{aligned}
$$

This can be written as

$$
\left[\begin{array}{l}
V_{1} \\
I_{1}
\end{array}\right]=[F]\left[\begin{array}{l}
V_{2} \\
I_{2}
\end{array}\right]
$$

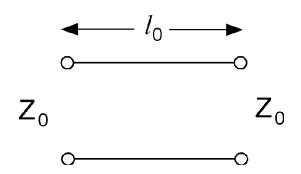

Fig. 4. Equivalent transmission line circuit representation of the straight waveguide section (C).

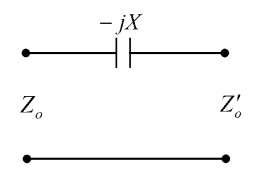

Fig. 5. Equivalent transmission line representation of the junction (B) between the straight waveguide segment and the $E$-plane bend.

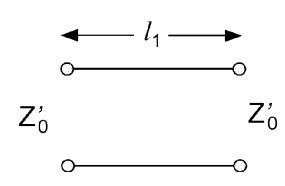

Fig. 6. Equivalent transmission line circuit representation of an $E$-plane bend (A).

where

$$
F=\left[\begin{array}{cc}
\cos \kappa & j Z \sin (\kappa) \\
j Y \sin (\kappa) & \cos (\kappa)
\end{array}\right]
$$

Here, $V_{2}$ is the voltage (which represents an electric field) at the input of a section of the waveguide (usually half of a physical period), $V_{1}$ is the voltage at the output, $\kappa$ is the phase shift, and $Y$ and $Z$ are the admittance and impedance of the FW equivalent circuit.

Meanwhile, the individual components of the cascaded matrix model of the waveguide (i.e., the straight section, the bend, the hole, etc) can be treated as individual transmission line segments [12]. Specifically the straight waveguide section (C) can be modeled as a uniform section of transmission line of length $l_{0}$ and characteristic impedance $Z_{0}=K_{\mathrm{wg}, 10}$, where $K_{\mathrm{wg}, 10}$ is the $\mathrm{TE}_{10}$ waveguide transverse mode impedance of (9). The equivalent transmission line circuit representation of the uniform section is shown in Fig. 4.

The straight-waveguide-to-E-plane-bend junction (B) can be represented by the equivalent transmission line circuit of Fig. 5.

Expressions for the parameters in Fig. 5 are given as [11]

$$
\begin{aligned}
& \frac{Z_{o}^{\prime}}{Z_{o}}=1+\frac{1}{12}\left(\frac{b}{R^{2}}\right)\left[\frac{1}{2}-\frac{1}{5}\left(\frac{2 \pi b}{\lambda_{g}}\right)^{2}\right] \\
& \frac{X}{Z_{o}}=\frac{32}{\pi^{7}}\left(\frac{2 \pi b}{\lambda_{g}}\right)^{3}\left(\frac{b}{R}\right)^{2} \sum_{n=1,3, \ldots}^{\infty} \frac{1}{n} \sqrt{1-\left(\frac{2 b}{n \lambda_{g}}\right)^{2}}
\end{aligned}
$$

$a$ and $b$ are the waveguide cross-sectional dimensions, $R$ is the bend radius, and $\lambda_{g}$ is the wavelength of the wave in the waveguide.

An equivalent transmission line circuit for the $E$-plane bend (A) is given in Fig. 6.

Here $l_{1}=1 / 2 \pi R$ is the length of the $E$-plane bend. 


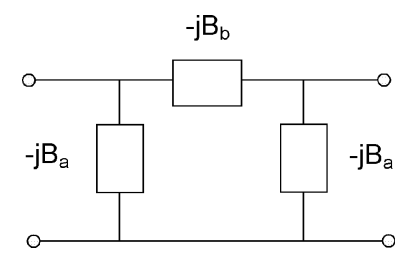

Fig. 7. Circuit model of three-way junction of rectangular and circular waveguide.

Finally, a simple circuit model of the (cutoff) beam hole (D) was not available from [11]. Therefore, the circuit equivalent was obtained from a modification of the components of a similar structure provided in [11]. The reference structure is a circular waveguide joined orthogonally to the broad wall of a rectangular waveguide through a small aperture. The difference between the reference structure and the structure to be modeled is that the circular tunnel of the structure to be modeled is a stub whose diameter equals the aperture diameter and is below the cutoff for propagation and there are two of these stubs present. These differences lead to a modification of the circuit equations of the reference structure. The circuit model of the reference structure [11] is shown in Fig. 7.

The equations for the components $B_{a}$ and $B_{b}$ are [11]

$$
\begin{aligned}
& \frac{B_{b}}{Y_{o}}=\frac{\lambda_{g} a b}{4 \pi}\left[\frac{1}{M}-\left(\frac{\pi}{a^{2} b}+\frac{7.74}{2 \pi R^{3}}\right)\right] \\
& \frac{B_{a}}{Y_{o}}=\frac{\frac{2 \pi P}{\lambda_{g} a b}\left(\frac{\lambda_{g}}{\lambda}\right)^{2}}{1-\frac{4 \pi P}{\lambda^{2} b}}
\end{aligned}
$$

where $Y_{0}$ is the admittance of the rectangular waveguide, $\lambda_{g}$ is the waveguide wavelength, $a$ and $b$ are the waveguide dimensions, $R$ is the circular waveguide radius (in this case $R=d$ as the aperture is the same size as the circular waveguide/beam tunnel), and $\lambda$ is the freespace wavelength. $M$ and $P$ are

$$
\begin{gathered}
M=\frac{d^{3}}{6} \\
P=\frac{d^{3}}{12}
\end{gathered}
$$

where $d$ is the aperture diameter.

To modify the above equations for the structure to be modeled, we observed that the aperture is the same size as the circular tunnel (which is cutoff). Hence, the fringing fields that normally exist on both sides of the aperture, and contribute to the shunt admittance $B_{b}$, only exist on one side. Therefore, we chose to halve the value of the circuit component $B_{b}$. However, because there are two holes and not one, all component values were doubled. This leaves us with the original value of $B_{b}$ and twice the original value of $B_{a}$.

As discussed above, the cascaded product of these individual circuit components can be equated to the single equivalent transmission line matrix $[F]$

$$
\left[\begin{array}{cc}
\cos \kappa & j Z \sin (\kappa) \\
j Y \sin (\kappa) & \cos (\kappa)
\end{array}\right]=[A][B][C][D][C][B][A]
$$

Since the right-hand side of (17) can be calculated from the circuit equivalent models of each of the components, we can solve for $\kappa$, which is the effective phase advance of the wave as

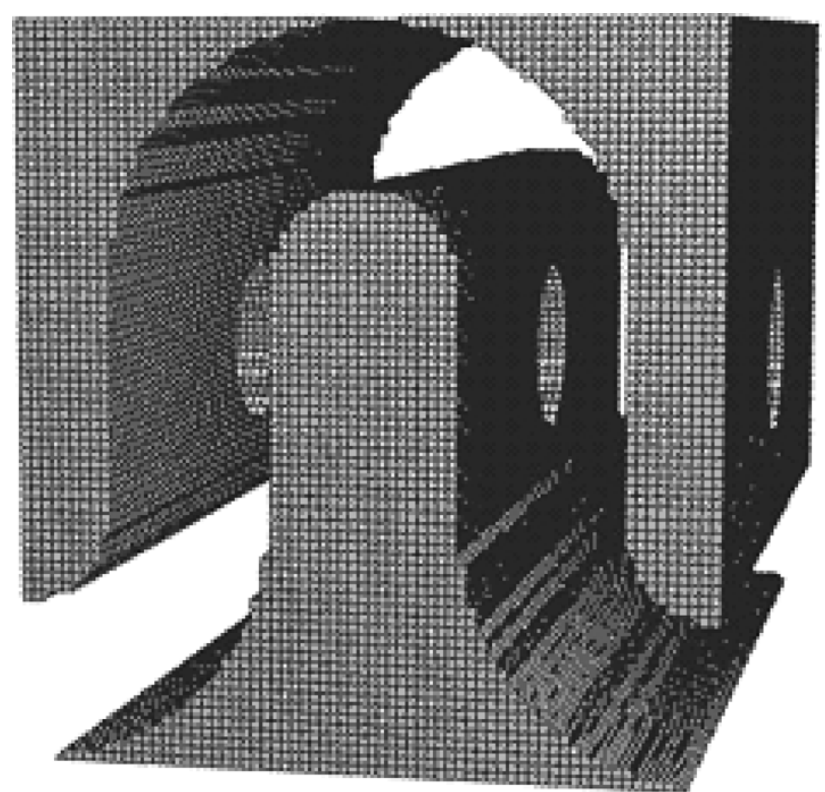

Fig. 8. Three-dimensional cutaway view of simulated FW circuit (maximum mesh $=300000$ cells) using MAFIA.

"seen" by the electron beam down the axis of that one section of FW.

Using a half-period of the structure, the effective axial propagation constant is therefore calculated from

$$
\beta_{z}=\frac{\pi+\kappa}{p}
$$

where $p$ is the pitch, or length of half of a period of the FW. The extra $\pi$ term accounts for the inherent $180^{\circ}$ phase shift of the electric field with respect to the electrons, due to the folded nature of the waveguide.

The phase velocity as seen by the electrons is then

$$
v_{p}=\frac{\omega}{\beta_{z}} .
$$

\section{MAFIA}

The Solution of Maxwells equations by the finite-integration-algorithm (MAFIA) is a 3-D, electromagnetic, particle-in-cell (PIC) code. The finite integration technique (FIT) algorithm produces a matrix of finite-difference equations for electric and magnetic field vectors in the structure under study. The solution of these equations yields static, frequency-domain or time-domain solutions of Maxwell's equations. The cold-test (beam free) dispersion is calculated using MAFIA by simulating a single geometrical period, or $2 p$, of the structure as shown in Fig. 8. As done previously for helical circuits in [13], the MAFIA eigenmode solver is used to apply quasi-periodic boundary conditions at the longitudinal ends permitting the user to choose a fixed phase shift per turn $\beta_{0} p$ in the axial direction. This allows the frequency to be obtained at any axial phase shift, and the corresponding phase velocity to be calculated using (1). The on-axis interaction impedance is then calculated at this phase shift directly from (2), where $\left|E_{z, m}\right|$ is obtained by doing a spatial Fourier analysis on the total on-axis axial electric field, and the time averaged RF power flow $P_{\mathrm{wg}}$ 
is calculated by integrating the Poynting vector over the circuit cross-section.

MAFIA has the option of automatically defining the mesh based on the user's input of a maximum number of mesh cells. The code then discretizes the geometry into rectangular and triangular cells, keeping the mesh spacing as uniform as possible. For the modeled structure consisting of $2 p$, we found that the phase velocity converged for a maximum mesh of 300000 cells. The impedance is less sensitive to the mesh spacing converging with a maximum mesh of 50000 .

\section{HFSS}

Ansoft HFSS is a commercial software program that calculates the electromagnetic response of a structure in the frequency domain. Using the finite element method it can calculate $S$-parameters, near- and far-fields, eigenmodes of a structure, etc. The mesh used by HFSS is an adaptive mesh consisting of nonregular tetrahedral elements. The program automatically refines the mesh, when and where it is necessary, to achieve a user-specified accuracy. In a method similar to MAFIA, a phase shift across the structure is specified and an associated "eigenfrequency" is determined. Phase velocity and interaction impedance are determined using the same techniques and equations used when simulating with MAFIA.

\section{E. Microwave Studio}

CST Microwave Studio (MWS) [14] is a specialized tool for the solution of 3-D electromagnetic high-frequency problems. To suit a variety of applications, the software contains four different simulation techniques: a transient solver, a frequency-domain solver, an eigenmode solver, and a modal analysis solver. It utilizes the new perfect boundary approximation (PBA) technique to avoid stair-step approximations associated with other rectangular meshing techniques. With the PBA, the simulated structure and the electromagnetic fields are mapped to a hexagonal mesh, so it allows a good approximation of even curved surfaces within the cuboid mesh cells [14]. MWS generates an automatic mesh based on the user's specification of frequency range, and mesh-per-free-space-wavelength. For a structure of $2 p$ in length, we found that the phase velocity converged for a mesh-per-free-space-wavelength of about 80 at $40 \mathrm{GHz}$, which translates to a total volumetric mesh of about 120000 cells. Similar to the MAFIA results, the impedance was fairly insensitive to the mesh spacing. Fig. 9(a) shows a contour plot of the surface currents at about $45 \mathrm{GHz}$. The plot demonstrates that the inside bends have the strongest surface currents, and thus care must be taken that these regions are accurately represented with the mesh. The mesh spacing is also shown in Fig. 9(b).

\section{F. Experimental Methods}

Phase velocities were measured on an experimental circuit, nominally designed for FWTWT operation in the 40-55-GHz range [1]. The measurements used a conventional technique of drawing a conductive bead on the end of a dielectric rod along the axis of the circuit while launching a wave into one end of the FW circuit. The magnitude of the reflected wave was recorded as a function of position of the bead, producing a standing wave
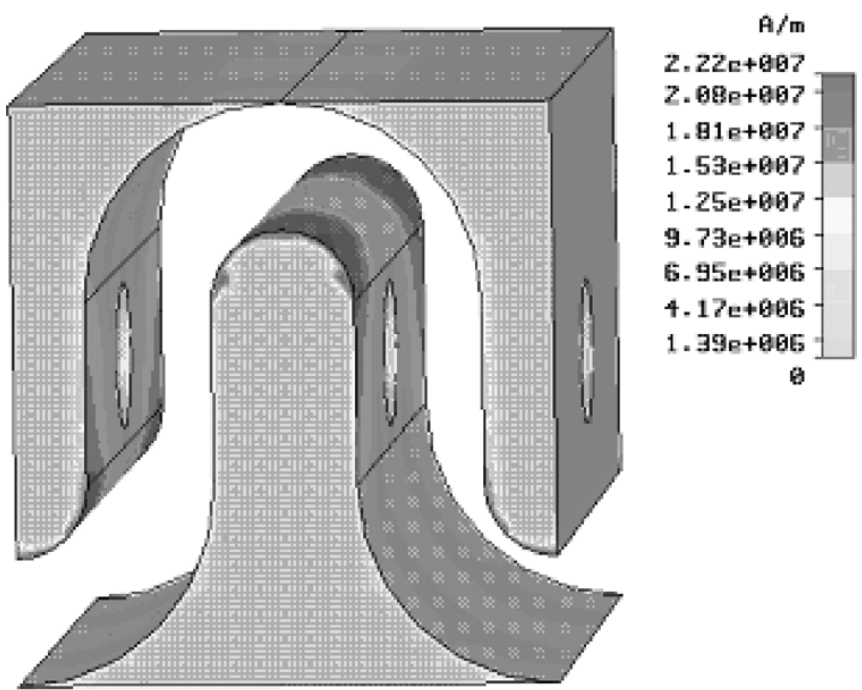

(a)

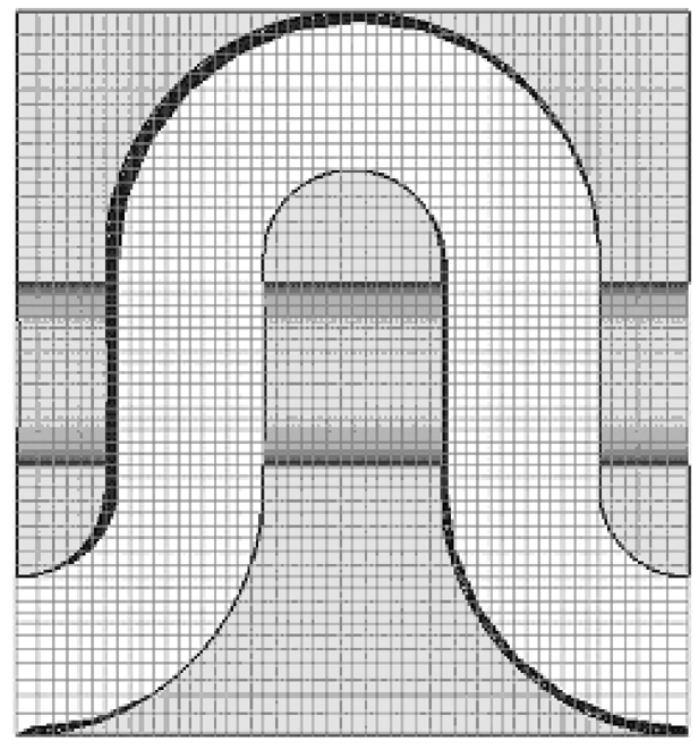

(b)

Fig. 9. Simulated contour plot of (a) surface currents and (b) side view of mesh using MWS.

pattern. In the frequency range of interest to this paper, this method is known to produce very accurate determinations of the effective axial wavenumber, from which the phase velocity can be directly calculated. In particular, the largest error would be bounded by the measurement of the distance between nodes on the standing wave plot. Based on a position measurement accuracy of less than $0.5 \mathrm{~mm}$, the error in the measured phase velocity values is $<0.2 \%$.

Small-signal gain was measured with thermistor detectors and a Hewlett Packard 431 power meter. Input power was supplied with an extended interaction oscillator (EIO).

\section{RESUlTS AND DISCUSSION}

\section{A. Phase Velocity}

Figs. 10 and 11 compare experimental measurements against the various models. Good agreement between measurements and model predictions was obtained for the circuit model, 


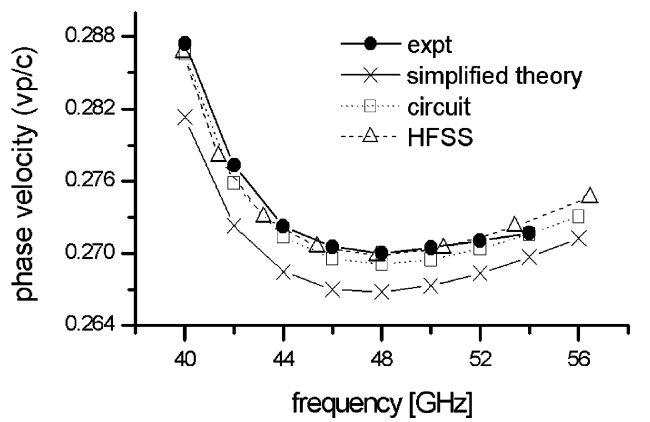

Fig. 10. Plot of effective axial phase velocity versus frequency, comparing experimental measurements, simplified theory, equivalent circuit, and HFSS models.

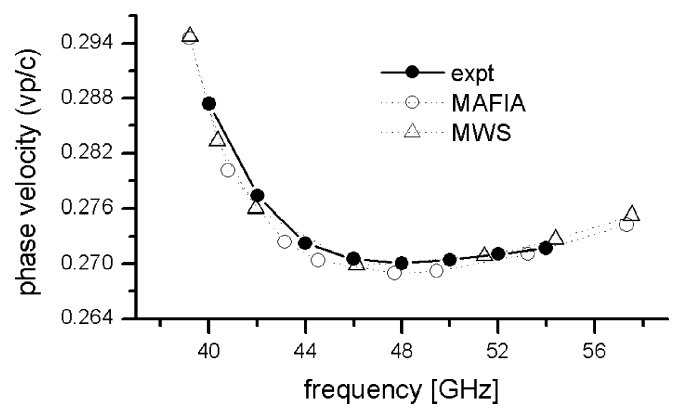

Fig. 11. Plot of effective axial phase velocity versus frequency, comparing experimental measurements, and MAFIA and MWS models.

HFSS [4], MWS, and MAFIA, with a sufficiently resolved computational grid. For example, a computational mesh of 50000 points in the MAFIA simulations was found to be inadequate. As discussed previously, it took approximately 300000 mesh cells in the MAFIA simulations to arrive at phase velocity measurements in good agreement with the experimental data. 300000 mesh points corresponded to approximately 115 cells-per-free-space-wavelength at $40 \mathrm{GHz}$. The MWS simulations used 120000 grid cells, corresponding to approximately 80 cells-per-free-space-wavelength at $40 \mathrm{GHz}$. For the HFSS computations, a convergence specification of $0.05 \%$ on the eigenfrequency was used.

As can be seen, the simplified theory underestimates the effective axial phase velocity, by $1 \%-2 \%$ over the gain bandwidth. Subsequently, it will be shown that the sensitivity to phase velocity of this high space charge device requires a more accurate estimation of velocity and therefore, the simplified theory is not sufficiently accurate.

In contrast, all three of the 3-D electromagnetic code predictions replicated the experimental results to better than $0.5 \%$ accuracy when sufficient grid resolution or convergence specifications were used (see above discussion).

Of the models that accurately calculate the phase velocity, the circuit model seems to be one of the most accurate. Also, since it is analytic, it is easier to work into an optimization algorithm and is faster to compute. This is true, however, only when the device modeled has a small beam hole. The accuracy of the circuit model decreases as the beam hole radius increases and for large beam hole radii the circuit model is not sufficiently accurate. This point is illustrated in Figs. 12 and 13. For small beam radii, the circuit model compares favorably to the HFSS model.

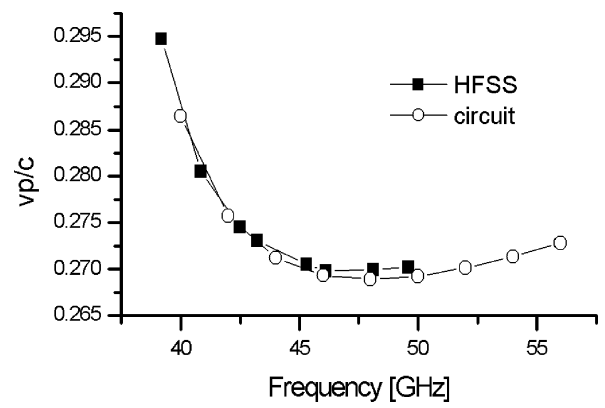

Fig. 12. Plot of effective axial phase velocity versus frequency, comparing equivalent circuit and HFSS results for a FWTWT with a small beam hole radius: $r_{c} / b=57.6 \%$.

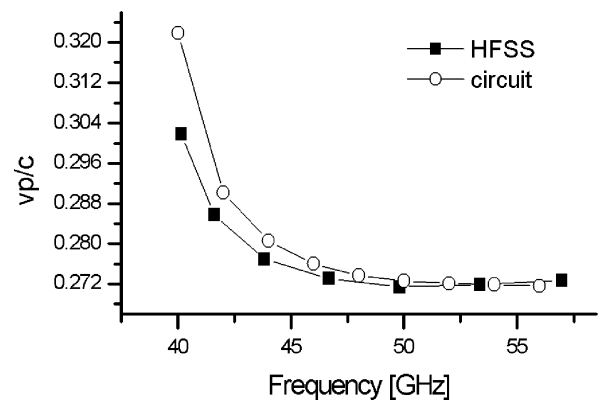

Fig. 13. Plot of effective axial phase velocity versus frequency, comparing equivalent circuit and HFSS results for a FWTWT with a large beam hole radius: $r_{c} / b=98.8 \%$.

For larger beam hole radii, the circuit model estimates a significantly higher phase velocity than the HFSS model.

Based on all examinations to date, the circuit model accuracy is acceptable, i.e., it predicts phase velocity to better than $0.5 \%$, comparable to the 3-D numerical code (HFSS in this case) for $r_{c} / b<\sim 85 \%$. Here, $r_{c}$ is the circuit radius and $b$ is the small waveguide dimension.

The difference between the circuit and 3-D numerical code models in Fig. 13 is most likely due to the choice of the equivalent circuit used to represent the beam hole. (see Section II-B) That is, this particular model appears to be accurate for small beam holes but suffers from loss of accuracy when $r_{c} / b \geq \sim 1$. A more accurate solution might be obtained from using the same equivalent circuit model, but modestly and empirically adjusting the values of various coefficients using an adaptive algorithm (e.g., as in [16]) to obtain an improved fit for large as well as small hole diameters. Another alternative would be to examine the application of cutoff beam hole models for klystron cavities [17].

\section{B. Interaction Impedance}

Fig. 14 compares the on-axis interaction impedance of various models. As can be seen, there is fairly good agreement between all the models. The greatest variation in estimated impedance seems to be at the lower band edge, and even there the difference is less than $13 \%$ between any model and the mean value.

\section{Small and Large Signal Gain}

The experimental small signal and saturated gain are compared to the output of CHRISTINE1D [6]. CHRISTINE1D is a 


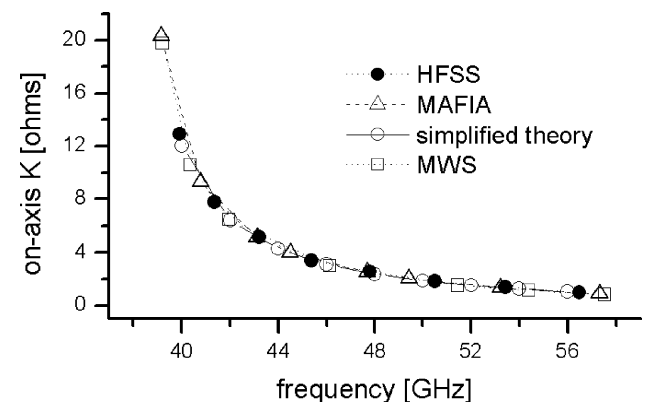

Fig. 14. Plot of on-axis interaction impedance vversus frequency, comparing various models.

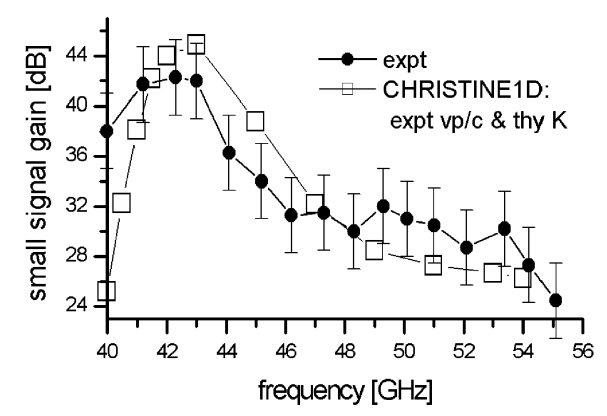

Fig. 15. Plot of small signal gain versus frequency, comparing experimental measurements with CHRISTINE1D TWT model using experimentally determined effective axial phase velocity and the beam-averaged interaction impedance from the simplified theory model.

1-D physical model, which uses phase velocity and beam-averaged interaction impedance to estimate the gain of a TWT. The beam-averaged interaction impedance can be obtained by multiplying the on-axis interaction impedance by the factor $I_{0}^{2}\left(\kappa_{\mathrm{cm}} r_{b}\right)-I_{1}^{2}\left(\kappa_{\mathrm{cm}} r_{b}\right)$ where $r_{b}$ is the beam radius and $\kappa_{\mathrm{cm}}$ is defined in (10) [18]. $I_{0}$ and $I_{1}$ are modified Bessel functions. For the simulations the beam voltage, current (unsaturated) and fill factor $\left(r_{b} / r_{c}\right)$ were $21.2 \mathrm{kV}, 200 \mathrm{~mA}$, and 0.5 , respectively, consistent with the experimental parameters [1], [10]. The experiment included a magnetic confinement factor of 1.5 (above Brillioun), ensuring good beam confinement. Although the experimental device did not include a sever, it did use a graphite attenuator between the input and output sections [1], [10]. The effects of this attenuator were incorporated into the CHRISTINE1D simulations, based on measurements of the frequency-dependent loss.

Fig. 15 compares the experimental small signal gain with the corresponding prediction from CHRISTINE1D. The agreement is remarkably within $4 \mathrm{~dB}$ across the entire band from $41-54 \mathrm{GHz}$, except at the very low band edge point at $40 \mathrm{GHz}$ (discussed further below). Figs. 16 and 17 show the sensitivity of small signal gain calculations to variations in phase velocity and interaction impedance. As can be seen from the plots, the predictions are extremely sensitive to the specification of phase velocity, but significantly less sensitive to the specification of interaction impedance. In particular, varying the phase velocity by as little as $0.5 \%$ results in changes of up to $8 \mathrm{~dB}$ in the predicted small signal gain. In contrast, a $10 \%$ variation in the interaction impedance results in a more modest $5 \mathrm{~dB}$ change in the predicted small signal gain.

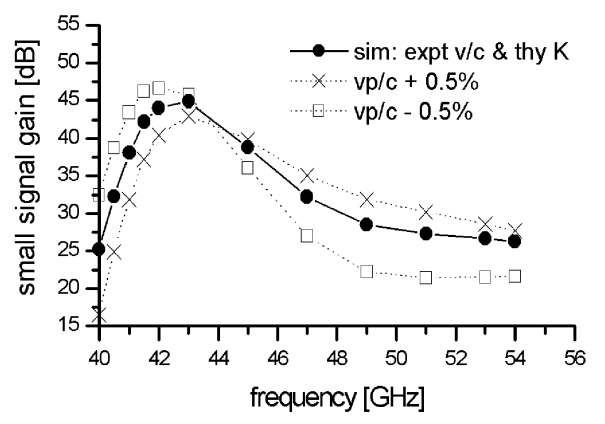

Fig. 16. Plot of small signal gain versus frequency as beam-averaged phase velocity is varied by $\pm 0.5 \%$.

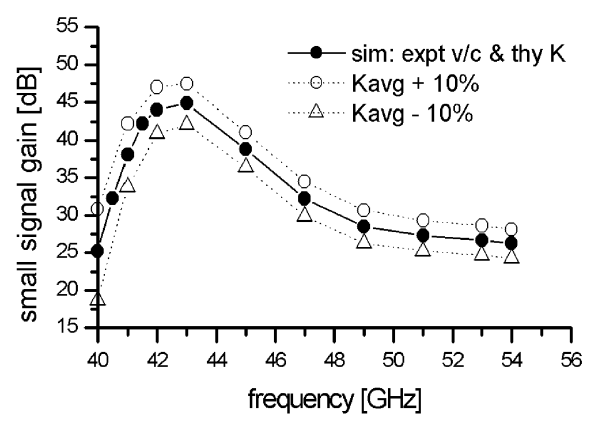

Fig. 17. Plot of small signal gain versus frequency as beam-averaged interaction impedance is varied by $\pm 10 \%$.

There are several explanations for the discrepancy of a much higher experimental than predicted small signal gain at the low-frequency band edge. The experimental gain was determined from measurements of output power using a broadband detector, (the power meter). Hence, it is possible that the experimental values are higher than predicted due to unfiltered second harmonic power. However, this possibility is unlikely, both because the measurements were taken under small signal conditions and because the beam-wave velocity mismatch is considerable and the coupling impedance small near $80 \mathrm{GHz}$. A more plausible explanation is that $40 \mathrm{GHz}$ is near the waveguide cutoff frequency for the circuit $(\sim 38 \mathrm{GHz})$. This, combined with (or responsible for) coupler mismatch could lead to extra feedback (frequency-dependent input-output coupler mismatch effects were not included in the simulations). Such extra feedback, while insufficient for start oscillation, would be capable of enhancing the measured gain above the theoretical single-pass prediction.

Fig. 18 compares CHRISTINE1D predictions of saturated output power to experimental measurements. The agreement is good, being exact near $42 \mathrm{GHz}$ and within $4 \mathrm{~dB}$, even at the low frequency band edge at $40 \mathrm{GHz}$. Experimental data at higher frequencies were not available due to the lack of a power source at higher frequencies that was able to saturate the TWT.

The sensitivity of the saturated performance to phase velocity and interaction impedance was also examined. Table I summarizes the results. In general, it was found that the sensitivity of the saturated output power and saturated gain were approximately equivalent when specified in decibels. As observed with the small-signal gain, the accuracy of the phase velocity has a much more sensitive effect on the predicted saturated power or gain than the accuracy of the interaction impedance. However, 


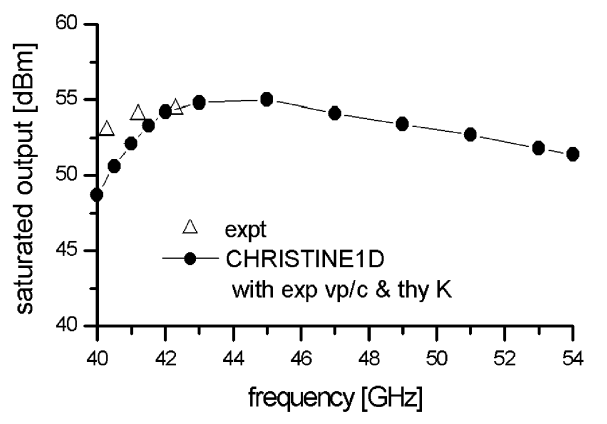

Fig. 18. Plot of saturated gain versus frequency, comparing experimental measurements with CHRISTINE1D TWT model using experimentally determined effective axial phase velocity and the beam-averaged interaction impedance from the simplified theory model.

TABLE I

EFFECT OF INPUT PARAMETER ERRORS ON PREDICTED SATURATED POWER OR GAIN

\begin{tabular}{c|c|c}
\hline Input & $\begin{array}{c}\text { parameter } \\
\text { error }\end{array}$ & $\begin{array}{c}\text { Saturated } \\
\text { power or } \\
\text { gain error }\end{array}$ \\
\hline Phase velocity error & $+0.5 \%$ & $+2-3 \mathrm{~dB}$ \\
\cline { 2 - 3 } & $-0.5 \%$ & $-3-4 \mathrm{~dB}$ \\
\hline $\begin{array}{c}\text { Interaction impedance } \\
\text { error }\end{array}$ & $+10 \%$ & $+1-2 \mathrm{~dB}$ \\
\cline { 2 - 3 } & $-10 \%$ & $-1-3 \mathrm{~dB}$ \\
\hline
\end{tabular}

the sensitivity of the saturated power or gain is noticeably less than (approximately half) the small-signal gain to either parameter. For example, a $10 \%$ error in the interaction impedance can result in a 5-dB error in the small-signal gain, but only a $1-3-\mathrm{dB}$ error in the predicted saturated power or gain. Similarly, a $0.5 \%$ error in the cold circuit phase velocity can result in as much as an $8-\mathrm{dB}$ error in the predicted small signal gain, but only a $2-4-\mathrm{dB}$ error in the predicted saturated power or gain.

Given these accuracy constraints on phase velocity and interaction impedance, it is interesting to examine what physical features of the circuits have the greatest influence on both parameters. It is straightforward to derive the following approximate expressions for $v_{p}\left(\omega_{0}\right)$ and $K\left(\omega_{0}\right)$ (the latter evaluated at the edge of the beam hole) from a simplified model of the circuit. Here, $\omega_{0}$ is the frequency at which $v_{p}$ is a minimum, which is nominally in the "middle" of the operating band of the FWTWT. While these expressions may not provide sufficient quantitative accuracy to meet the $0.5 \%$ and $10 \%$ guidelines for phase velocity and impedance, respectively, they nevertheless provide useful scaling properties to understand design sensitivity effects. Specifically, for the phase velocity

$$
\frac{v_{p}\left(\omega_{0}\right)}{c} \approx \frac{\frac{p}{a}}{\sqrt{1+\frac{L^{2}}{a^{2}}}}
$$

and the interaction impedance

$$
\left.K\left(\omega_{0}\right)\right|_{r=r_{c}} \approx 47.5 \frac{\frac{b}{L}}{\left(1+\frac{L^{2}}{a^{2}}\right)^{3 / 2}} .
$$

The parameters are defined in Fig. 2. Note, then, that the phase velocity is directly proportional to the pitch, $p$, as expected, and the interaction impedance is proportional to the small cross-sectional dimension of the waveguide, $b$. Therefore, any errors in $p$ or $b$, will lead directly to proportionate errors in $v_{p}$ or $K$, respectively. The factor $\left(1+L^{2} / a^{2}\right)^{1 / 2}$ happens to be equal to the frequency ratio $\omega_{0} / \omega_{\text {co }}$, where $\omega_{\text {co }}$ is the cutoff frequency of (6). For the circuit used for these studies, $\omega_{0} / \omega_{\text {co }}=1.25$.

\section{SUMMARY AND CONCLUSION}

From this paper, we have established that accurate, full-bandwidth predictions of output power or gain of FWTWTs are possible with 1-D parametric TWT models if phase velocity and interaction impedance functions are sufficiently well characterized. The most sensitive performance parameter was observed to be the small signal gain. It was observed that predictions of the small signal gain to within 4-dB of experimental measurements on a 40-55-GHz FWTWT could be realized over virtually the entire operating band. Similar agreement was observed for saturated gain over a limited frequency band for which experimental data was available.

Sensitivity studies indicate that variations in the phase velocity of $0.5 \%$ can result in $8 \mathrm{~dB}$ of variation in the predicted small-signal gain, while a $10 \%$ variation in the interaction impedance can result in a $5-\mathrm{dB}$ change in the predicted small signal gain. The small signal gain prediction is approximately twice as sensitive to variations in these input parameters as the saturated power or saturated gain. Based on these observations, it is indicated that the use of parametric 1-D TWT models for accurate, full-band predictions of small-signal gain in FWTWTs requires knowledge of phase velocity and impedance functions that are accurate to $<0.5 \%$ and $<10 \%$, respectively. Saturated gain predictions, being approximately half as sensitive to these parameters, would ostensibly require correct specification of phase velocity and interaction impedance to within $\sim 1 \%$ and $20 \%$ respectively.

An equivalent transmission line circuit model for the FW structure was observed to predict phase velocity very well for smaller beam holes but poorly for large holes, while 3-D electromagnetics codes were observed to predict phase velocity functions that yielded highly accurate TWT gain predictions (e.g., within $4 \mathrm{~dB}$ across the band of an experimental test device). To obtain the required accuracy on phase velocity, it was necessary to implement aggressive constraints on convergence or mesh resolution. For example, using the MAFIA simulation code, a mesh spacing of approximately 115 cells-per-free-space-wavelength of linear dimension was required. With MWS, a mesh of approximately 80 cells-per-free-space-wavelength of linear dimension at $40 \mathrm{GHz}$ was suitable. With the HFSS finite element code in eigenmode solver mode, a convergence specification of $0.05 \%$ on the eigenfrequency was found acceptable. The simplified analytic model was not acceptable. All methods examined successfully predicted the interaction impedance to within the required $10 \%$ tolerance.

\section{REFERENCES}

[1] G. Döhler, D. Gagne, D. Gallagher, and R. Moats, "Serpentine waveguide TWT," in IEDM Tech. Dig., 1987, pp. 485-488.

[2] R. G. E. Hutter, Beam and Wave Electronics in Microwave Tubes. New York: Van Nostrand, 1960. 
[3] R. G. Carter, W. Bosch, V. Srivastava, and G. Gatti, "Computer simulation of intermodulation distortion in traveling wave tube amplifiers," IEEE Trans. Electron Devices, vol. 48, no. 1, pp. 178-180, Jan. 2001.

[4] HFSS High Frequency Structure Simulator, Ansoft Corporation, Los Angeles, CA.

[5] MAFIA. Computer Simulation Technology (CST) GmbH. [Online]. Available: www.cst.de

[6] T. Antonsen, Jr. and B. Levush, "CHRISTINE: A multifrequency parametric simulation code for traveling wave tube amplifiers," Naval Res. Lab., Washington, DC, NRL/FR/6840-97-9845.

[7] H. P. Freund, E. G. Zaidman, and T. M. Antonsen, Jr., "Theory of helix traveling wave tubes with dielectric and vane loading," Phys. Plasmas, vol. 3, no. 8, Aug. 1996.

[8] J. H. Booske, "New opportunities in vacuum electronics through the application of microfabrication technologies," in Proc. Int. Vacuum Electronics Conf., Apr. 2002.

[9] S. Bhattacharjee et al., "Folded waveguide traveling wave tube sources for THz radiation," in IEEE Trans. Plasma Sci., vol. 32, May 2004, pp. 1002-1014.

[10] G. Döhler, D. Gallagher, and J. Richards, Proc. Vac. Elec. Ann. Rev., Crystal City, VA, 1993, pp. V15-V20.

[11] N. Marcuvitz, Waveguide Handbook. Stevenage, U.K.: Peregrinus, 1986.

[12] J. J. Choi, C. M. Armstrong, A. K. Ganguly, and F. Calise, "Folded waveguide gyrotron traveling-wave-tube amplifier," Phys. Plasmas, vol. 2, no. 3, Mar. 1995.

[13] C. L. Kory and J. A. Dayton, Jr., "Computational investigation of experimental interaction impedance obtained by perturbation for helical traveling-wave tube structures," IEEE Trans. Electron Devices, vol. 45, no. 9, pp. 2063-2071, Sep. 1998.

[14] Microwave Studio. Computer Simulation Technology (CST) GmbH. [Online]. Available: www.cst.de

[15] J. W. Maruschek, C. L. Kory, and J. D. Wilson, "Generalized three-dimensional simulation of ferruled coupled-cavity traveling-wave tube dispersion and impedance characteristics," NASA, Greenbelt, MD, NASA TP-3389, 1993.

[16] P. L. Werner, R. Mittra, and D. H. Werner, "Extraction of equivalent circuits for microstrip components and discontinuities using the genetic algorithm," IEEE Microw. Guided Wave Lett., vol. 8, no. 4, pp. 333-335, Apr. 1998.

[17] K. Fujisawa, "General treatment of Klysron resonant cavities," IRE Trans. Microw. Theory Tech., vol. MTT-6, pp. 344-358, 1958.

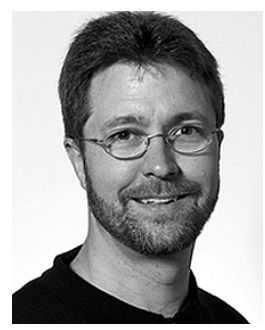

John H. Booske (SM'93) received the Ph.D. degree in nuclear engineering from the University of Michigan, Ann Arbor, n 1985

He was a Research Scientist with the University of Maryland, College Park, from 1985 to 1989. In 1990 , he joined the faculty of the University of Wisconsin, Madison, where he is a Professor of Electrical and Computer Engineering. In 2001, he was appointed Director of the Materials Science Program. His research interests include the experimental and theoretical study of coherent electromagnetic radiation sources and their applications, with emphasis in the RF, microwave, millimeter-wave, and tetrahertz regimes. Recent research activities include vacuum electronics, microwave and RF heating of materials, and biological/biomedical applications of microwave and RF fields. He is coeditor of the book Microwave and Radio Frequency Applications (Westerville, OH: American Ceramic Society, 2003) and coeditor of the book Microwave and Millimeter-Wave Power Electronics (Piscataway, NJ: IEEE Press, 2004).

Dr. Booske has received the University of Wisconsin Vilas Associate Faculty Award for research excellence and the U.S. National Science Foundation Presidential Young Investigator Award. He has been honored with many teaching awards, including the University of Wisconsin Chancellor's Distinguished Teaching Award, and the Benjamin Smith Reynolds Award for Excellence in Teaching Engineering. He served as Co-Director of a U.S. DoD MURI99 Consortium on Innovative Microwave Vacuum Electronics, and as Director of a MURI04 Consortium on cathode field emission and RF window breakdown in high-power microwave sources. He has been a Guest Editor of the IEEE TRANSACTIONS ON PLASMA SCIENCE.

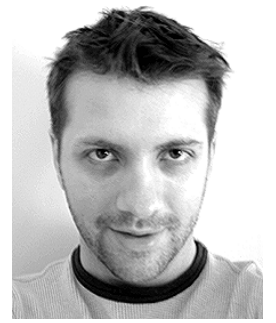

Mark C. Converse (S'00) received the B.S., M.S., and Ph.D. degrees in electrical engineering from the University of Wisconsin, Madison, in 1996, 1999, and 2003 , respectively.

During his graduate studies, he was engaged in plasma processing research involving damage evaluation/analysis and mitigation during the etching process. In 1999, he began research in microwave vacuum electronics investigating the impulse response of the helix-TWT. Currently he is working as a Postdoctoral Scholar at the Department of Electrical and Computer Engineering, University of Wisconsin on projects involving the microwave detection and treatment of breast and liver cancer using microwaves. His research interests include electromagnetic interactions with materials, electrical/biological interfaces, and organic electronics.

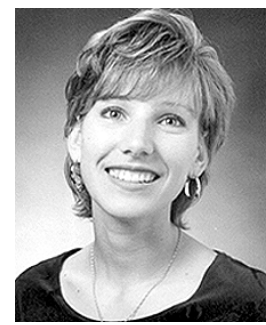

Carol L. Kory (SM'00) received the B.S.E.E degree from the University of Dayton, Dayton, OH, in 1992 , and the M.S.E.E and Ph.D. degrees in engineering from Cleveland State University, Cleveland, $\mathrm{OH}$, in 1997 and 2000, respectively

From 1991 to 1992, she was with the NASA Glenn Research Center, Cleveland, as an intern student with the Communication Technology $\mathrm{Di}$ vision, where her work involved analyzing TWT slow-wave circuits using a 3-D particle-in-cell code. She became a full-time employee of the Analex Corporation in 1992, working with the same staff at NASA Glenn. Her work predominantly involves computational modeling, design, and development of vacuum electronics devices.

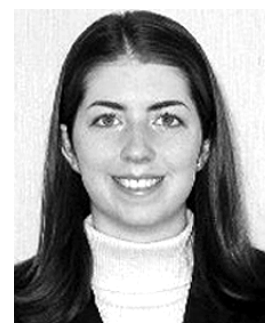

Christine T. Chevalier (S'00) received the B.S degree in electrical engineering from the University of Dayton, Dayton, OH, in 2001 and is currently pursuing the M.S. degree in electrical engineering at Cleveland State University, Cleveland, OH.

Upon graduation from the University of Dayton, she became a full-time employee of the Analex Corporation, working at the NASA Glenn Research Center, Cleveland. Her research interests include computational modeling and the design/optimization of 3-D electromagnetic circuits.

Ms. Chevalier is a member of Tau Beta Pi.

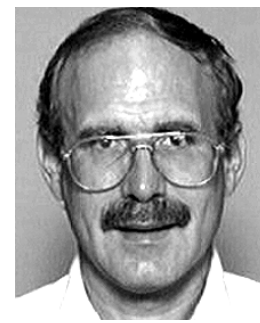

David A. Gallagher received the Ph.D. degree in physics from the University of Illinois at Urbana-Champaign.

He has since worked with the vacuum electronics research group at Northrop Grumman Corporation, Rolling Meadows, IL, where he has performed theoretical studies and simulations, has worked on the design and development of slow-wave, fast-wave, microwave, and millimeter-wave electron tubes, including peniotrons, orotrons, FTWTs, cusp electron guns for high harmonic gyro-device applications, and printed planar circuit TWTs using CNT cold-cathodes, and has recently expanded into electro-optical Infrared (EO/IR) systems. 


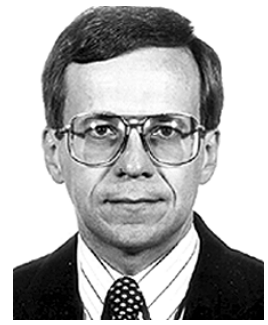

Kenneth E. Kreischer (M'00) received the B.S. degree in physics and the Ph.D. degree in nuclear engineering from the Massachusetts Institute of Technology (MIT) Cambridge, in 1976 and 1981, respectively.

He remained at MIT from 1981 to 2000, where he worked as a Principal Research Scientist at the MIT Plasma Science and Fusion Center. His main responsibility at MIT was overseeing the experimental research activities of the Waves and Beam Division. A major area of research was the design and testing of MW gyrotron oscillators operating at $100-300 \mathrm{GHz}$ suitable for plasma heating applications. Other responsibilities included the development of a high gradient accelerator operating at $17 \mathrm{GHz}$, an RF photocathode gun capable of producing a high brightness electron beam, and submillimeter instrumentation used to increase the sensitivity of EPR and NMR spectrometers. He joined Northrop Grumman Corporation, Rolling Meadows, IL, in 2000, and is presently Director of vacuum electronics technology. His present research interests include highfrequency amplifiers and tunable sources for radar and imaging, microwave power modules, $\mathrm{THz}$ instrumentation, quasi-optical mode converters and transmission line components, and field emission electron sources. He has authored over 60 publications on these subjects.

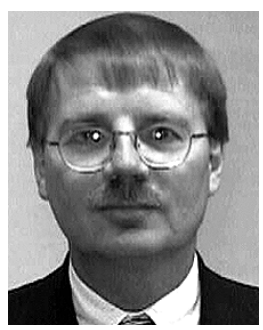

Vernon O. Heinen (M'97) received the B.S. degree from the University of Minnesota, Minneapolis, in 1977, and the M.S. and Ph.D. degrees from Michigan State University (MSU), East Lansing, in 1979 and 1983, respectively, both in physics, and the M.E. degree in electrical engineering from the University of Utah, Salt Lake City, in 1986. His graduate school work at MSU was in low-temperature solid-state physics.

After graduation, he joined the Microwave Amplifier Section at NASA Glenn Research Center, Cleveland, $\mathrm{OH}$, developing vacuum electronic devices for space communication applications. He served as Manager of Communications Technology during a temporary assignment at NASA Headquarters, Washington, DC, in 1988. On returning to Glenn Research Center he worked to develop space applications of high-temperature superconducting materials and field-emitting cathodes for microwave applications. In 1998, he became the Chief of the Electron Device Technology Branch. He joined Northrop Grumman Corporation, Rolling Meadows, IL, in 2000 as Director of Vacuum Electronics Devices.

Dr. Heinen is a member of the American Physical Society.

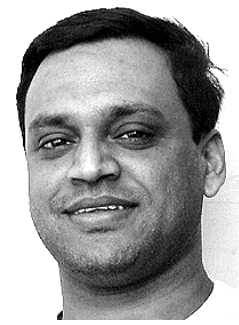

Sudeep Bhattacharjee (M'00) received the B.S. and M.S. degrees in physics from the University of North Bengal, Bengal, India, in 1989 and 1992, respectively, and the Ph.D. degree in experimental plasma physics on a Japanese Government Ministry of Education, Science, and Culture (Monbusho) scholarship from the Institute of Physical and Chemical Research (RIKEN), Saitama, Japan, in affiliation with Saitama University, in 1999.

He was at the Institute of Plasma Research (IPR) first as a Graduate Research Scholar from 1993 to 1994 and then as a Scientific Officer from 1995 to 1996, under the Department of Atomic Energy, Government of India. From 1999 to 2001 he was a Distinguished Postdoctoral Fellow (Kisotokken) at the cyclotron center of RIKEN where he did pioneering work involving development of a high-power microwave plasma ion source for nuclear physics applications such as a generation of heavy ions and multicharged radioactive ion beams. He joined the University of Wisconsin (UW), Madison as a Postdoctoral Research Associate in October 2001. His research at UW primarily involves development of electromagnetic sources of tetrahertz radiation and the generation and control of chaos in TWT amplifiers for communication and radar applications. He was selected to the physics faculty at the Indian Institute of Technology, Kanpur, India, in November, 2003.

Dr. Bhattacharjee is a member of the American Physical Society, the Physical Society of Japan, and the Japan Society of Applied Physics. He received the IEEE Early Career Travel Grant Award at the International Microwave Symposium (IMS'03) in Philadelphia. 